\title{
Hawking radiation from cubic and quartic black holes via tunneling of GUP corrected scalar and fermion particles
}

\author{
Wajiha Javed, ${ }^{1, *}$ Rimsha Babar, ${ }^{1, \dagger}$ and Ali Övgün ${ }^{2,3, \ddagger}$ \\ ${ }^{1}$ Division of Science and Technology, University of Education, Township-Lahore, Pakistan \\ ${ }^{2}$ Instituto de Física, Pontificia Universidad Católica de Valparaíso, Casilla 4950, Valparaíso, Chile \\ ${ }^{3}$ Physics Department, Faculty of Arts and Sciences, Eastern Mediterranean University, Famagusta, North Cyprus, via Mersin 10, Turkey
}

(Dated: December 18, 2018)

\begin{abstract}
We analyze the effect of the generalized uncertainty (GUP) principle on the Hawking radiation from the hairy black hole in U(1) gauge-invariant scalar-vector-tensor theory by utilizing the semiclassical Hamilton-Jacobi method. To do so, we evaluate the tunneling probabilities and Hawking temperature for scalar and fermion particles for the given spacetime of the black holes with cubic and quartic interactions. For this purpose, we utilize the modified Klein-Gordon equation for the Boson particles and then Dirac equations for the fermion particles, respectively. Next, we examine that the Hawking temperature of the black holes do not depend on the properties of tunneling particles. Moreover, we present the corrected Hawking temperature of scalar and fermion particles looks similar in both interactions, but there are different mass and momentum relationships for scalar and fermion particles in cubic and quartic interactions.
\end{abstract}

PACS numbers: 04.20.Jb, 04.62.+v, 04.70.Dy

Keywords: Effective Hawking temperature, Modified Klein-Gordon, Modified Dirac equation.

\section{INTRODUCTION}

As indicated by Einstein's theory of general relativity, black holes (BHs) are very intense transcendental objects that twist spacetime so unequivocally that no light or matter can get out of their grasp. According to NASA, some primordial BHs have shaped before long after the Big Bang and may be of the estimate of a single molecule, anyhow, as enormous as a mountain. In 1960's, John Wheeler and his collaborators propounded that BHs "have no hair", an allegory meaning that BHs trim of all complex features. In general relativity, no-hair theorem postulates that BHs are surprisingly simple objects, they can only describe by three exceptional classical parameters for them: their mass, electromagnetic charge and angular momentum [1]-[5]. The Wheelers hypothesis of no hair theorem was proven wrong in Einstein-Yang-Mills theory [6]. It is familiar that BHs have hair within the existence of Yang-Mills fields [7]-[9].

The modification of the general relativity may include scalar fields. In these theories no-hair theorem still exists. The primary no-scalar-hair theorem has connected to the massless scalar [10] and to the impartial scalar fields with a monotonous increasing self-inducting potential [11]. Heisenberg and Sujikawa [12] investigated the solution of hairy $\mathrm{BH}$ under U(1) gauge-invariant scalar-vector-tensor (SVT) theories for cubic and quartic order scalar-vector interactions. Herdeiro and Radu [13] have introduced a new family of BHs with scalar hair, that's persistently associated to the Kerr family and gave a subjectively new case of hairy BHs entitled Kerr BHs with scalar hair. It is familiar that BHs can grow scalar hair in the presence of matter in their proximity [14, 15], complex scalar with time dependent stages [16]-[18], or on the off chance that when the asymptotics are cosmological or anti-de Sitter [19]-[21].

General relativity extensions to generalized gravitation theories extensively presents new parametric quantities apart from two tensor polarization [22]. The development of SVT theories with Horndeski theories was also carried out for both the U(1) gauge and non-gauge invariant cases [23]. The new parametric quantities emerging in SVT theories are important for the thermodynamical properties of BHs and expansion of universe.

Stephen Hawking (1970), presented his idea about black body radiations, which is known as Hawking radiation. All BHs losses their mass as apparitional quantum particles [24, 25]. Due to the continuous process of Hawking radiation a $\mathrm{BH}$ evaporates [26, 27]. Hawking evaluated the $\mathrm{BH}$ physics in a curved spacetime, under quantum field theory depending upon the Heisenberg uncertainty principle (GUP). The Hawking radiation through quantum

\footnotetext{
*Electronic address: wajiha.javed@ue.edu.pk; wajihajaved84@yahoo.com

${ }^{\dagger}$ Electronic address: rimsha.babar10@gmail.com

‡Electronic address: ali.ovgun@pucv.cl; https : / /www. aovgun.com
} 
tunneling strategy of the radiated particles from a $\mathrm{BH}$ has been investigated [28]-[31]. Moreover, the Hawking radiation through tunneling phenomenon for numerous $\mathrm{BHs}$ has been investigated, abundantly, in $(2+1)$ and $(3+$ 1)-dimensional [32-86]. Many authors have studied quantum tunneling approach for particles with different spins such as vectors (bosons), scalar and fermions through the horizons of various BHs, wormholes and other celestial objects, they also calculated their corresponding Hawking temperatures.

The GUP plays very important role to evaluate the quantum gravity effects (quantum corrections). To acquire the GUP effects on Hawking temperature, we will utilize corrections of Klein-Gordan and Dirac equations by considering quantum effects. Black holes are the main experimentation field to investigate the quantum gravity effects and lots of literature on $\mathrm{BH}$ thermodynamical properties to study the quantum gravity effects under GUP. The BH thermodynamics have moreover been explored within the system of GUP [87]-[93]. Nozari and Mehdipour [94] have investigated the tunneling phenomenon under GUP effects for Schwarzschild BH and also evaluated its modified tunneling rate. Övgün et al. [95] have calculated the tunneling of massive spin- 1 and spin-0 particles for a warped Dvali-Gabadadze-Porrati (DGP) gravity BH and also discussed the effects of GUP for both type of particles. Övgün, Javed and Ali [96] have found the tunneling rate of charged massive bosons for various types of BHs surrounded by the perfect fluid in Rastall theory. Sharif and Javed [97] have considered tunneling phenomenon for fermion particles through the event horizons for the study of Hawking temperature. They also discussed the Hawking radiation through tunneling phenomenon for fermion particles from a pair of charged accelerating and rotating $\mathrm{BH}$ around NUT parameter [98]. Furthermore, they analyzed the corresponding Hawking temperature of these BHs. Sharif and Javed [99] have also investigated the quantum corrections for regular BHs, i.e., Bardeen and ABGB.

In the continuation of the previous work, we will investigate the quantum corrections of massive scalar and fermion particles by using GUP effects. A brief outline of paper is given as follows: In Section II, we provide the detail of hairy $\mathrm{BH}$ in the existence of U(1) gauge scalar-vector-tensor theories. In Section III, we explore the GUP-corrected Klein-Gordan equation for cubic and quartic interactions and examine the quantum corrections of Hawking temperature for massive scalar particles for BH solution surrounded by SVT theory. Whereas, Section IV is devoted to the same analysis with Dirac equation for fermion particles. Finally, the results of this paper are summarized in Section V.

\section{HAIRY BLACK HOLE UNDER GAUGE-INVARIANT SCALAR-VECTOR-TENSOR THEORY}

Black holes play a very essential role in general relativity. The Gibbons solution which depicts a ReissnerNordstorm $\mathrm{BH}$ with a non-trivial expansion field is considered as the first hairy BHs. Whereas numerous ways run out scalar field hair in asymptotically flat spacetime [100]. However, more hairy BHs have gotten in models motivated by string theory and counting expansion, curvature correction and many other. By considering positive or negative, cosmological constant asymptotically (anti)-de-Sitter hairy BHs are obtained. Hairy BHs generally occur in natural models. However, large hairy BHs are regularly unstable and during perturbation they loose their hair, although the stable one are exceptionally little. The hairy BHs yields as cosmologically large, which conflicts the observation [101]. It will be very interesting to evaluate the tunneling probability and corrected Hawking temperature from such a $\mathrm{BH}$. The metric of hairy $\mathrm{BH}$ for cubic and quartic interactions is defined as [12]

$$
d s^{2}=-Z(r) d t^{2}+h^{-1}(r) d r^{2}+r^{2}\left(d \theta^{2}+\sin ^{2} \theta d \phi^{2}\right),
$$

where for cubic interaction

$$
\begin{gathered}
Z(r)=1-\frac{2 M}{r}+\frac{Q^{2}}{2 M_{p l}^{2} r^{2}}+\frac{3 \beta_{3}^{2} Q^{4}}{14 M_{p l}^{2} r^{8}}+O\left(\frac{1}{r^{9}}\right), \\
h(r)=1-\frac{2 M}{r}+\frac{Q^{2}}{2 M_{p l}^{2} r^{2}}-\frac{2 \beta_{3}^{2} Q^{4}}{7 M_{p l}^{2} r^{8}}+O\left(\frac{1}{r^{9}}\right) .
\end{gathered}
$$

For quartic interaction

$$
\begin{aligned}
Z(r) & =1-\frac{2 M}{r}+\frac{Q^{2}}{2 M_{p l}^{2} r^{2}}-\frac{2 \beta_{4} Q^{2}}{M_{p l}^{2} r^{4}}+\frac{2 \beta_{4} M Q^{2}}{M_{p l}^{2} r^{5}}-\frac{3 \beta_{4} Q^{4}}{5 M_{p l}^{4} r^{6}}+\frac{256 \beta_{4}^{2} M Q^{2}}{7 M_{p l}^{2} r^{7}} \\
& +\frac{3 Q^{2}\left(M_{p l}^{2} Q^{2} \beta_{3}^{2}-28 \beta_{4}^{2} Q^{2}-256 \beta_{4}^{2} M^{2} M_{p l}^{2}\right)}{14 M_{p l}^{4} r^{8}}, \\
h(r) & =1-\frac{2 M}{r}+\frac{Q^{2}}{2 M_{p l}^{2} r^{2}}-\frac{2 \beta_{4} M Q^{2}}{M_{p l}^{2} r^{5}}+\frac{2 \beta_{4} Q^{4}}{5 M_{p l}^{4} r^{6}}-\frac{2 Q^{2}\left(\beta_{3}^{2} Q^{2}-64 \beta_{4}^{2} M^{2}\right)}{7 M_{p l}^{2} r^{8}} .
\end{aligned}
$$


Here, $M$ stands for $\mathrm{BH}$ mass and $Q$ represents the charge of $\mathrm{BH}$.

\section{SCALAR PARTICLE TUNNELING VIA MODIFIED KLEIN-GORDAN EQUATION}

This section is based on the effects of GUP on the tunnling of massive scalar particles from the hairy BH solutions for cubic and quartic interactions.

\section{A. Cubic Interaction for Scalar Particles}

This section is devoted to study the tunneling phenomenon of scalar particles for given $\mathrm{BH}$ with cubic interaction and analyze the corresponding corrected Hawking temperature. For this purpose, the modified Klein-Gordan equation is given by [102]

$$
-(\breve{\hbar})^{2} \partial^{t} \partial_{t} \Phi=\left[(-\iota \breve{\hbar})^{2} \partial^{i} \partial_{i}+m_{0}^{2}\right]\left[1-2 \breve{\beta}(-\iota \breve{\hbar})^{2} \partial^{i} \partial_{i}+m_{0}^{2}\right] \breve{\Phi},
$$

The wave function $\breve{\Phi}$ for scalar field is given as

$$
\breve{\Phi}(t, r, \theta, \varphi)=\left[\frac{\imath}{\breve{h}} I(t, r, \theta, \varphi)\right],
$$

we consider just first order term in $\breve{h}$, so the above Eq.(21) becomes

$$
\begin{aligned}
& \frac{1}{Z(r)}\left(\partial_{t} I\right)^{2}=\left[h(r)\left(\partial_{r} I\right)^{2}+\frac{1}{r^{2}}\left(\partial_{\theta} I\right)^{2}+\frac{1}{r^{2} \sin ^{2} \theta}\left(\partial_{\varphi} I\right)^{2}+m_{0}^{2}\right] \times \\
& {\left[1-2 \breve{\beta}\left(h(r)\left(\partial_{r} I\right)^{2}+\frac{1}{r^{2}}\left(\partial_{\theta} I\right)^{2}+\frac{1}{r^{2} \sin ^{2} \theta}\left(\partial_{\varphi} I\right)^{2}+m_{0}^{2}\right)\right] .}
\end{aligned}
$$

To solve this equation, the particle's action is defined as

$$
I=-E t+W(r, \theta)+j \varphi .
$$

Here, $W(r, \theta)$ cannot be parted as $W(r) \Theta(\theta)$. For sake of simplicity, we can fix the angle $\theta$ at a particular value of $\theta_{0}$. At $\theta=\theta_{0}$, Eq.(5) gets the following form

$$
\breve{A}\left(\partial_{r} W\right)^{4}+\breve{B}\left(\partial_{r} W\right)^{2}+\breve{C}=0,
$$

here

$$
\begin{aligned}
& \breve{A}=-2 \breve{\beta} h^{2}(r), \quad \breve{B}=h^{2}(r)\left(1-\frac{4 \breve{\beta} j^{2}}{r^{2} \sin ^{2} \theta}-4 \breve{\beta} m_{0}^{2}\right), \\
& \breve{C}=m_{0}^{2}+\frac{j^{2}}{r^{2} \sin ^{2} \theta}-\frac{2 \breve{\beta} j^{4}}{r^{4} \sin ^{4} \theta}-\frac{4 \breve{\beta} m_{0}^{2} j^{2}}{r^{2} \sin ^{2} \theta}-2 \breve{\beta} m_{0}^{4}-\frac{E^{2}}{Z(r)} .
\end{aligned}
$$

After solving Eq.(7), we get

$$
\begin{aligned}
W_{ \pm}(r) & = \pm \int \frac{d r}{\sqrt{Z(r)}}\left[1+\breve{\beta}\left(m_{0}^{2}+\frac{E^{2}}{Z(r)}+\frac{j^{2}}{r^{2} \sin ^{2} \theta}\right)\right] \\
& \times \sqrt{E^{2}-Z(r)\left(m_{0}^{2}-\frac{j^{2}}{r^{2} \sin ^{2} \theta}\right)-2 \breve{\beta} Z(r)\left(\frac{j^{4}}{r^{4} \sin ^{4} \theta}+2 m_{0}^{2} Z(r) \frac{j^{2}}{r^{2} \sin ^{2} \theta}+m^{4}\right)} .
\end{aligned}
$$

After ignoring higher order terms of $\breve{\beta}$, we solve above integral to calculate the imaginary part of function at event horizon

$$
\operatorname{ImW}\left(r_{+}\right)= \pm \pi\left(\frac{E r_{+}^{2}}{\Delta_{r}\left(r_{+}\right)}\right)(1+\breve{\beta} \Xi)
$$


where

$$
\Xi=\left(m^{2}+\frac{E^{2}}{F^{\prime}\left(r_{+}\right)}+\frac{j^{2} \csc ^{2} \theta}{r_{+}^{2}}\right),
$$

Here, $W_{+}$and $W_{-}$are the radial functions for the outgoing and incoming particles, respectively. The tunneling probability for scalar particles at $r=r_{+}$is given as follows

$$
\begin{aligned}
\breve{\Gamma} & =\frac{\breve{\Gamma}_{(\text {out })}}{\breve{\Gamma}_{(\text {in })}}=\frac{\exp \left[-\frac{2}{\grave{\hbar}}\left(\operatorname{Im} W_{+}\right)\right]}{\exp \left[-\frac{2}{\hbar}\left(I m W_{-}\right)\right]}=\exp \left[-\frac{4}{\breve{\hbar}} \operatorname{Im} W_{+}\right], \\
& =\exp \left[-\frac{4 \pi r_{+}^{2}}{\breve{\hbar} \Delta_{, r}\left(r_{+}\right)}(E) \times(1+\breve{\beta} \Xi)\right] .
\end{aligned}
$$

For $\breve{\hbar}=1$ and utilizing Boltzmann factor $\breve{\Gamma}=\exp \left(\frac{E}{T_{H}^{\prime}}\right)$, the modified temperature can be obtained as

$$
T_{H}^{\prime}=\frac{\Delta_{, r}\left(r_{+}\right)}{4 \pi r_{+}^{2}(1+\breve{\beta} \Xi)}=T_{H}(1-\breve{\beta} \Xi)
$$

where

$$
T_{H}=\frac{1}{4 \pi r_{+}^{2}}\left[\frac{2 M}{r_{+}^{2}}-\frac{Q^{2}}{M_{p l}^{2} r_{+}^{3}}-\frac{12 Q^{4} \beta_{3}^{2}}{7 M_{p l}^{2} r_{+}^{9}}-O\left(\frac{1}{r_{+}^{10}}\right)\right],
$$

which is the original Hawking temperature of a corresponding $\mathrm{BH}$.

\section{B. Quartic Interaction for Scalar Particles-I}

Following the same procedure given in the preceding subsection $\mathbf{A}$, for this line element (2), we can calculate the corrected Hawking temperature with the effects of quantum gravity for this $\mathrm{BH}$. The modified Hawking temperature for quartic interactions can be deduced as

$$
T_{H}^{\prime}=\frac{\Delta_{, r}\left(r_{+}\right)}{4 \pi r_{+}^{2}(1+\breve{\beta} \Xi)}=T_{H}(1-\breve{\beta} \Xi)
$$

where the original Hawking temperature is

$$
\begin{aligned}
T_{H} & =\frac{1}{4 \pi r_{+}^{2}}\left[\frac{2 M}{r_{+}^{2}}-\frac{Q^{2}}{M_{p l}^{2} r_{+}^{2}}-\frac{256 M Q^{2} \beta_{4}^{2}}{M_{p l}^{2} r_{+}^{8}}-\frac{10 \beta_{4} M Q^{2}}{M_{p l}^{2} r^{6}}+\frac{8 \beta_{4} Q^{2}}{M_{p l}^{2} r^{5}}+\frac{18 \beta_{4} Q^{4}}{5 M_{p l}^{4} r^{7}}\right. \\
& \left.-\frac{12 Q^{2}\left(M_{p l}^{2} Q^{2} \beta_{3}^{2}-28 \beta_{4}^{2} Q^{2}-256 \beta_{4}^{2} M^{2} M_{p l}^{2}\right)}{7 M_{p l}^{4} r^{9}}\right] .
\end{aligned}
$$

\section{Quartic Interaction for Scalar Particles-II}

The line element of $\mathrm{BH}$ for Quartic interactions is defined as

$$
d s^{2}=-Z(r) d t^{2}+h^{-1}(r) d r^{2}+r^{2}\left(d \theta^{2}+\sin ^{2} \theta d \breve{\Phi}^{2}\right),
$$

where

$$
\begin{aligned}
& Z(r)=(1-\mu)\left(\frac{r}{r_{+}}-1\right)-\frac{1-2 \mu+12 \beta_{3}^{2} \mu^{2}(1-\mu)+8 \bar{\beta}_{4} \mu(1-2 \mu)}{1+8 \bar{\beta}_{4}}\left(\frac{r}{r_{+}}-1\right)^{2} \\
& h(r)=(1-\mu)\left(\frac{r}{r_{+}}-1\right)-\frac{1-2 \mu-4 \beta_{3}^{2} \mu^{2}(1-\mu)+8 \bar{\beta}_{4} \mu(1-2 \mu)}{1+8 \bar{\beta}_{4}}\left(\frac{r}{r_{+}}-1\right)^{2} .
\end{aligned}
$$


where $\tilde{\beta}_{3}=\frac{\beta_{3} M_{p l}^{2}}{r_{+}^{2}}$ and $\bar{\beta}_{4}=\frac{\beta_{4} M_{p l}^{2}}{r_{+}^{4}}$.

Using the same formalism as defined earlier in (A) for this line element, we obtain the corrected Hawking temperature with the effect of quantum gravity for this $\mathrm{BH}$. The modified Hawking temperature for quartic interactions is deduced as

$$
T_{H}^{\prime}=\frac{\Delta_{, r}\left(r_{+}\right)}{4 \pi r_{+}^{2}(1+\breve{\beta} \Xi)}=T_{H}(1-\breve{\beta} \Xi)
$$

where the original Hawking temperature is

$$
\begin{aligned}
T_{H} & =\frac{1}{r_{+}^{3}\left(r_{+}^{4}+8 M_{p l}^{2} \beta_{4}\right)^{2}}\left[1 6 \mu M _ { p l } ^ { 2 } r _ { + } \left(-3(\mu-1) \mu M_{p l}^{2} \beta_{3}^{2}\right.\right. \\
& \left.+2(1-2 \mu) \beta_{4}\right)\left(r-r_{+}\right)^{2}\left(r_{+}^{4}+8 M_{p l}^{2} \beta_{4}\right)-32 M_{p l}^{2} \beta_{4} r_{+}^{4}(1-2 \mu)\left(r-r_{+}\right) \\
& -4 \mu M_{p l}^{2} r_{+}\left(-3(\mu-1) \mu M_{p l}^{2} \beta_{3}^{2}+2(1-2 \mu) \beta_{4}\right)-r_{+}(\mu-1)\left(r-r_{+}\right) \\
& \times\left(r_{+}^{4}+8 M_{p l}^{2} \beta_{4}\right)^{2}-2 r_{+}^{2}\left(r_{+}^{4}+8 M_{p l}^{2} \beta_{4}\right)(1-2 \mu)-4 \mu M_{p l}^{2}\left(r-r_{+}\right) \\
& \left.\times\left(-3(\mu-1) \mu M_{p l}^{2} \beta_{3}^{2}+2(1-2 \mu) \beta_{4}\right)\right] .
\end{aligned}
$$

\section{THE FERMION PARTICLES TUNNELING VIA MODIFIED DIRAC EQUATION}

In this section, we will foccus on studying the effects of GUP on the tunneling procedure of fermion particles from the hairy $\mathrm{BH}$ for cubic and quartic interactions.

\section{A. Cubic Interactions for Fermion Particles}

In order to investigate the quantum tunneling of fermion particles for hairy $\mathrm{BH}$, the Dirac equation is given as follows [74]

$$
i \tilde{\gamma}^{v}\left(\partial_{v}+\tilde{\Omega}_{v}\right) \Psi+\frac{m_{0}}{\hbar} \Psi=0, \quad v=0,1,2,3
$$

where

$$
\tilde{\Omega}_{v}=\frac{i}{2} \tilde{\rightarrow}_{\nu}^{\alpha \beta} \tilde{\Sigma}_{\alpha \beta}, \quad \tilde{\Sigma}_{\alpha \beta}=\frac{1}{4} i\left[\tilde{\gamma}^{\alpha}, \tilde{\gamma}^{\beta}\right], \quad\left\{\tilde{\gamma}^{\alpha}, \tilde{\gamma}^{\beta}\right\}=2 \tilde{\eta}^{\alpha \beta},
$$

here $\sim_{\nu}{ }_{\nu}^{\alpha \beta}$ represents the spin connection which can be defined by the following relation of tetrad $e^{\lambda}$, i.e.,

$$
\stackrel{\sim}{\rightarrow}_{\nu \beta}^{\alpha}=e_{\mu}^{\alpha} e_{\beta}^{\lambda} \tilde{\Gamma}_{v \lambda}^{\mu}-e_{\beta}^{\lambda} \partial_{\nu} e_{\lambda}^{\alpha},
$$

and the $\tilde{\gamma}^{v '}$ s in curved space-time can be defined as

$$
\tilde{\gamma}^{v}=e_{\alpha}^{v} \tilde{\gamma}^{\alpha}, \quad\left\{\tilde{\gamma}^{a}, \tilde{\gamma}^{b}\right\}=2 \tilde{g}^{a b},
$$

The modified Dirac equation is defined as

$$
-i \tilde{\gamma}^{0} \partial_{0} \tilde{\Psi}=\left(i \tilde{\gamma}^{i} \partial_{i}+i \tilde{\gamma}^{\mu} \tilde{\Omega}_{v}+\frac{m_{0}}{\tilde{\hbar}}\right)\left(1+\tilde{\beta} \tilde{\hbar}^{2} \partial_{j} \partial^{j}-\tilde{\beta} m_{0}^{2}\right) \tilde{\Psi},
$$

the above equation can be rewrite in the following form as

$$
\begin{aligned}
& {\left[i \tilde{\gamma}^{0} \partial_{0}+i \tilde{\gamma}^{i}\left(1-\tilde{\beta} m_{0}^{2}\right) \partial_{i}+i \tilde{\beta} \tilde{\hbar}^{2} \tilde{\gamma}^{i} \partial_{i}\left(\partial_{j} \partial^{j}\right)+\frac{m_{0}}{\tilde{\hbar}}\left(1+\tilde{\beta} \tilde{\hbar}^{2} \partial_{j} \partial^{j}-\tilde{\beta} m_{0}^{2}\right)\right.} \\
& \left.-i \tilde{\gamma}^{\mu} \tilde{\Gamma}_{\mu}\left(1+\tilde{\beta} \tilde{\hbar}^{2} \partial_{j} \partial^{j}-\tilde{\beta} m_{0}^{2}\right)\right] \tilde{\Psi}
\end{aligned}
$$


where $\tilde{\Psi}$ is generalized Dirac spinor. The Dirac gamma matrices $\gamma^{\nu \prime}$ s can be defined as

$$
\begin{array}{ll}
\tilde{\gamma}^{t}=\frac{1}{\sqrt{Z(r)}}\left(\begin{array}{cc}
i & 0 \\
0 & -i
\end{array}\right), & \tilde{\gamma}^{r}=\sqrt{h(r)}\left(\begin{array}{cc}
0 & \tilde{\sigma}^{3} \\
\tilde{\sigma}^{3} & 0
\end{array}\right), \\
\tilde{\gamma}^{\theta}=\sqrt{g^{\theta \theta}}\left(\begin{array}{cc}
0 & \tilde{\sigma}^{1} \\
\tilde{\sigma}^{1} & 0
\end{array}\right), & \tilde{\gamma}^{\phi}=\sqrt{g^{\varphi \varphi}}\left(\begin{array}{cc}
0 & \tilde{\sigma}^{2} \\
\tilde{\sigma}^{2} & 0
\end{array}\right),
\end{array}
$$

where $\sqrt{g^{\theta \theta}}=\frac{1}{r} \& \sqrt{g^{\varphi \varphi}}=\frac{1}{r \sin \theta}$ and Pauli sigma matrices $\tilde{\sigma}^{\prime}$ s can be expressed as follows

$$
\tilde{\sigma}^{1}=\left(\begin{array}{ll}
0 & 1 \\
1 & 0
\end{array}\right), \quad \tilde{\sigma}^{2}=\left(\begin{array}{cc}
0 & -i \\
i & 0
\end{array}\right), \quad \tilde{\sigma}^{3}=\left(\begin{array}{cc}
1 & 0 \\
0 & -1
\end{array}\right) .
$$

The wave functions can be defined as

$$
\tilde{\Psi}(t, r, \theta, \varphi)=\left[\begin{array}{c}
A(t, r, \theta, \varphi) \\
0 \\
B(t, r, \theta, \varphi) \\
0
\end{array}\right] \exp \left[\frac{i}{\hbar}(t, r, \theta, \varphi)\right],
$$

where $A(t, r, \theta, \varphi)$ and $B(t, r, \theta, \varphi)$ are arbitrary functions of spacetime coordinates.Using Eq.(21) and values of gamma matrices in Eq.(20), we get the following set of equations for first order in $\tilde{\hbar}$ and $\tilde{\beta}$, i.e.,

$$
\begin{aligned}
& -\frac{i A}{\sqrt{Z(r)}}\left(\partial_{t} I\right)-B \sqrt{h(r)}\left(1-\tilde{\beta} m_{0}^{2}\right)\left(\partial_{r} I\right)+B \tilde{\beta} \sqrt{h(r)}\left(\partial_{r} I\right)\left[h(r)\left(\partial_{r} I\right)^{2}+g^{\theta \theta}\left(\partial_{\theta} I\right)^{2}+g^{\varphi \varphi}\left(\partial_{\varphi} I\right)^{2}\right] \\
& -A m_{0} \tilde{\beta}\left[h(r)\left(\partial_{r} I\right)^{2}+g^{\theta \theta}\left(\partial_{\theta} I\right)^{2}+g^{\varphi \varphi}\left(\partial_{\varphi} I\right)^{2}\right]+\left(1-\tilde{\beta} m_{0}^{2}\right) A m_{0}=0, \\
& \frac{i B}{\sqrt{Z(r)}}\left(\partial_{t} I\right)-A \sqrt{h(r)}\left(1-\tilde{\beta} m_{0}^{2}\right)\left(\partial_{r} I\right)+B m_{0}\left(1-\tilde{\beta} m_{0}^{2}\right)+ \\
& A \beta \sqrt{h(r)}\left(\partial_{r} I\right)\left[h(r)\left(\partial_{r} I\right)^{2}+g^{\theta \theta}\left(\partial_{\theta} I\right)^{2}+g^{\varphi \varphi}\left(\partial_{\varphi} I\right)^{2}\right] \\
& -m_{0} B \tilde{\beta}\left[h(r)\left(\partial_{r} I\right)^{2}+g^{\theta \theta}\left(\partial_{\theta} I\right)^{2}+g^{\varphi \varphi}\left(\partial_{\varphi} I\right)^{2}\right]=0, \\
& B\left[-\sqrt{g^{\theta \theta}}\left(1-\tilde{\beta} m_{0}^{2}\right)\left(\partial_{\theta} I\right)-i\left(1-\tilde{\beta} m_{0}^{2}\right) \sqrt{g^{\varphi \varphi}}\left(\partial_{\varphi} I\right)+\tilde{\beta}\left(\partial_{\theta} I\right) \sqrt{g^{\theta \theta}}\right. \\
& \left\{h(r)\left(\partial_{r} I\right)^{2}+g^{\theta \theta}\left(\partial_{\theta} I\right)^{2}+g^{\varphi \varphi}\left(\partial_{\varphi} I\right)^{2}\right\}+i \tilde{\beta} \sqrt{g^{\varphi \varphi}}\left(\partial_{\varphi} I\right) \\
& \left.\left\{h(r)\left(\partial_{r} I\right)^{2}+g^{\theta \theta}\left(\partial_{\theta} I\right)^{2}+g^{\varphi \varphi}\left(\partial_{\varphi} I\right)^{2}\right\}\right]=0, \\
& A\left[-\sqrt{g^{\theta \theta}}\left(1-\tilde{\beta} m_{0}^{2}\right)\left(\partial_{\theta} I\right)-i\left(1-\tilde{\beta} m_{0}^{2}\right) \sqrt{g^{\varphi \varphi}}\left(\partial_{\varphi} I\right)+\tilde{\beta}\left(\partial_{\theta} I\right) \sqrt{g^{\theta \theta}}\right. \\
& \left\{h(r)\left(\partial_{r} I\right)^{2}+g^{\theta \theta}\left(\partial_{\theta} I\right)^{2}+g^{\varphi \varphi}\left(\partial_{\varphi} I\right)^{2}\right\}+i B \sqrt{g^{\varphi \varphi}}\left(\partial_{\varphi} I\right) \\
& \left.\left\{h(r)\left(\partial_{r} I\right)^{2}+g^{\theta \theta}\left(\partial_{\theta} I\right)^{2}+g^{\varphi \varphi}\left(\partial_{\varphi} I\right)^{2}\right\}\right]=0 .
\end{aligned}
$$

By using separation of variables technique, we assume

$$
\tilde{I}(t, r, \theta, \varphi)=-E t+W(r)+\Theta(\theta, \phi),
$$

where $E$ represents energy of the particle, and $J_{\theta}=\partial_{\theta} \Theta, J_{\phi}=\partial_{\phi} \Theta$.

Using Eq.(IV A) in Eqs.(22)-(25) and by focusing on Eqs.(24) and (25), we observe that they are homogeneous after dividing by $A$ and $B$. They can be rewritten as

$$
\begin{aligned}
& \left\{\tilde{\beta} h_{r}\left(r_{+}\right) W^{\prime 2}+g^{\theta \theta} \tilde{\beta} J_{\theta}^{2}+\tilde{\beta} g^{\varphi \varphi} J_{\varphi}^{2}-\left(1-\tilde{\beta} m_{0}^{2}\right)\right\} \\
& \times\left[\sqrt{g^{\theta \theta}} J_{\theta}+i \sqrt{g^{\varphi \varphi}} J_{\varphi}\right]=0
\end{aligned}
$$

here $W^{\prime}=\partial_{r} W, J_{\theta}=\partial_{\theta} \Theta$ and $\partial_{\varphi}=\partial_{\varphi} \Theta$. 
In Eq.(27), $\tilde{\beta}$ indicates the quantum gravity effects so it cannot be considered as zero, thus the term in large bracket equals to zero and provide the solution of $\Theta$. From Eq.(27), we can write the following expression

$$
\left[\sqrt{g^{\theta \theta}} J_{\theta}+i \sqrt{g^{\varphi \varphi}} J_{\varphi}\right]=0
$$

After removing $A$ and $B$ from Eqs.(22) and (23), we get homogeneous equations, which generate

$$
U_{6}\left(\partial_{r} W\right)^{6}+U_{4}\left(\partial_{r} W\right)^{4}+U_{2}\left(\partial_{r} W\right)^{2}+U_{0}=0,
$$

where

$$
\begin{aligned}
& U_{6}=\tilde{\beta}^{2} h^{3} Z, \\
& U_{4}=\tilde{\beta} h^{2} Z\left(m_{0}^{2} \tilde{\beta}+2 \tilde{\beta} S-2\right), \\
& U_{2}=h Z\left[\left(1-\tilde{\beta} m_{0}^{2}\right)^{2}+\tilde{\beta}\left(2 m_{0}^{2}-2 m_{0}^{4} \tilde{\beta}-2 S+\tilde{\beta} S^{2}\right)\right], \\
& U_{0}=m_{0}^{2}\left(1-\tilde{\beta} m_{0}^{2}-\tilde{\beta} S\right)^{2} Z-E^{2}, \\
& S=g^{\theta \theta} J_{\theta}^{2}+g^{\varphi \varphi} J_{\varphi}^{2} .
\end{aligned}
$$

From Eq.(28), we note that $S=0$. Considering $\beta$ only for first order and solving the above Eq.(29) at event horizon, which gives

$$
W(r)= \pm \int \frac{1}{\sqrt{h Z}} \sqrt{m_{0}^{2}\left(1-2 \tilde{\beta} m_{0}^{2}\right) Z+E^{2}}\left[1+\tilde{\beta}\left(m_{0}^{2}+\frac{E^{2}}{Z}\right)\right] d r
$$

The above equation implies

$$
W(r)= \pm i \pi \frac{E r_{+}^{2}}{\Delta_{r}\left(r_{+}\right)}(1+\tilde{\beta} \Sigma)
$$

where

$$
\Sigma=\left(m_{0}^{2}+\frac{E^{2}}{Z^{\prime}\left(r_{+}\right)}\right)
$$

The tunneling rate of scalar particles at $r=r_{+}$can be calculated as

$$
\begin{aligned}
\tilde{\Gamma} & =\frac{\tilde{\Gamma}_{(\text {out })}}{\tilde{\Gamma}_{(\text {in })}}=\frac{\exp \left[-\frac{2}{\tilde{\hbar}}\left(\operatorname{Im} W_{+}\right)\right]}{\exp \left[-\frac{2}{\tilde{\hbar}}\left(\operatorname{Im} W_{-}\right)\right]}=\exp \left[-\frac{4}{\tilde{\hbar}} \operatorname{Im} W_{+}\right], \\
& =\exp \left[-\frac{4 \pi r_{+}^{2}}{\tilde{\hbar} \Delta_{, r}\left(r_{+}\right)}(E) \times(1+\tilde{\beta} \Sigma)\right] .
\end{aligned}
$$

The modified Hawking temperature can be obtained as

$$
T_{H}^{\prime}=\frac{\Delta_{, r}\left(r_{+}\right)}{4 \pi r_{+}^{2}(1+\tilde{\beta} \Sigma)}=T_{H}(1-\tilde{\beta} \Sigma),
$$

where

$$
T_{H}=\frac{1}{4 \pi r_{+}^{2}}\left[\frac{2 M}{r_{+}^{2}}-\frac{Q^{2}}{M_{p l}^{2} r_{+}^{3}}-\frac{12 Q^{4} \beta_{3}^{2}}{7 M_{p l}^{2} r_{+}^{9}}-O\left(\frac{1}{r_{+}^{10}}\right)\right],
$$

which is the original Hawking temperature of a corresponding $\mathrm{BH}$. 


\section{B. Quartic Interaction for Fermion Particles-I}

Following the same process, for line element (2) of quartic interactions, we calculate the corrected Hawking temperature under the effect of quantum gravity. The modified Hawking temperature for quartic interactions of fermion particles is deduced as

$$
T_{H}^{\prime}=\frac{\Delta_{, r}\left(r_{+}\right)}{4 \pi r_{+}^{2}(1+\tilde{\beta} \Sigma)}=T_{H}(1-\tilde{\beta} \Sigma)
$$

where

$$
\begin{aligned}
T_{H} & =\frac{1}{4 \pi r_{+}^{2}}\left[\frac{2 M}{r_{+}^{2}}-\frac{Q^{2}}{M_{p l}^{2} r_{+}^{2}}-\frac{256 M Q^{2} \beta_{4}^{2}}{M_{p l}^{2} r_{+}^{8}}-\frac{10 \beta_{4} M Q^{2}}{M_{p l}^{2} r^{6}}+\frac{8 \beta_{4} Q^{2}}{M_{p l}^{2} r^{5}}+\frac{18 \beta_{4} Q^{4}}{5 M_{p l}^{4} r^{7}}\right. \\
& \left.-\frac{12 Q^{2}\left(M_{p l}^{2} Q^{2} \beta_{3}^{2}-28 \beta_{4}^{2} Q^{2}-256 \beta_{4}^{2} M^{2} M_{p l}^{2}\right)}{7 M_{p l}^{4} r^{9}}\right]
\end{aligned}
$$

which is the original Hawking temperature of the corresponding $\mathrm{BH}$.

\section{Quartic Interaction for Fermion Particles-II}

By following the same procedure, given in the preceding Section (A) for line element (13) of quartic interactions, we calculate the corrected Hawking temperature under the effect of quantum gravity. The modified Hawking temperature for quartic interactions of fermion particles is deduced as

$$
T_{H}^{\prime}=\frac{\Delta_{, r}\left(r_{+}\right)}{4 \pi r_{+}^{2}(1+\tilde{\beta} \Sigma)}=T_{H}(1-\tilde{\beta} \Sigma),
$$

where

$$
\begin{aligned}
T_{H} & =\frac{1}{r_{+}^{3}\left(r_{+}^{4}+8 M_{p l}^{2} \beta_{4}\right)^{2}}\left[-16 \mu M_{p l}^{2} r_{+}\left(-3(\mu-1) \mu M_{p l}^{2} \beta_{3}^{2}\right.\right. \\
& \left.+2(1-2 \mu) \beta_{4}\right)\left(r-r_{+}\right)^{2}\left(r_{+}^{4}+8 M_{p l}^{2} \beta_{4}\right)+32 M_{p l}^{2} \beta_{4} r_{+}^{4}(1-2 \mu)\left(r-r_{+}\right) \\
& +4 \mu M_{p l}^{2} r_{+}\left(-3(\mu-1) \mu M_{p l}^{2} \beta_{3}^{2}+2(1-2 \mu) \beta_{4}\right)+r_{+}(\mu-1)\left(r-r_{+}\right) \\
& \times\left(r_{+}^{4}+8 M_{p l}^{2} \beta_{4}\right)^{2}+2 r_{+}^{2}\left(r_{+}^{4}+8 M_{p l}^{2} \beta_{4}\right)(1-2 \mu)+4 \mu M_{p l}^{2}\left(r-r_{+}\right) \\
& \left.\times\left(-3(\mu-1) \mu M_{p l}^{2} \beta_{3}^{2}+2(1-2 \mu) \beta_{4}\right)\right] .
\end{aligned}
$$

which is the original Hawking temperature of the corresponding $\mathrm{BH}$.

\section{CONCLUSION}

In this work, we have analyzed the tunneling probability and corrected temperature $T_{H}^{\prime}$ of hairy $\mathrm{BH}$ for cubic and quartic interactions. For this purpose, we utilized Hamilton-Jacobi ansatz and WKB approximation and considered the modified Klein-Gordan and Dirac equations for scalar and fermion particles, respectively. We calculated corrected Hawking temperature for scalar and fermion particles and radiated temperature looked over preserved energy and charge. By utilizing modified Klein-Gordan and Dirac equations, the corrected Hawking temperature $T_{H}^{\prime}=T_{H}(1-\breve{\beta} \Xi)$ and $T_{H}^{\prime}=T_{H}(1-\breve{\beta} \Sigma)$ vales have been computed given in Eqs.(11) and (32) by considering the effects of quantum gravity. We have concluded that the quantum gravity effects increased the Hawking temperature. We demonstrated that the corrected Hawking temperatures does not just based on the properties of the $\mathrm{BH}$, yet in addition depend upon the quantum numbers, i.e., angular momentum, mass and energy of the transmitted particles. The expression of corrected Hawking temperature in Eqs.(11) and (12) for scalar particle look similar to the expressioin in Eq.(32) and (33) for fermion particles but the angular momentum and mass are not same. Moreover, the black holes with the quartic interactions have more Hawking temperature than $\mathrm{BH}^{\prime} \mathrm{s}$ with cubic interactions. We can conclude that the increasing number of interactions provide more radiation. 


\section{Acknowledgments}

A. Ö. acknowledges financial support provided under the Chilean FONDECYT Grant No. 3170035. The authors thank an anonymous referees for their useful suggestions that helped improve the paper.

[1] W. Israel, Phys. Rev. 164, 1776(1967).

[2] W. Israel, Commun. Math Phys. 8, 245(1968).

[3] B. Carter, Phys. Rev. Lett. 26, 331(1971).

[4] R. M. Wald, Phys. Rev. Lett. 26, 1653(1971).

[5] J. D. Bekenstein, Phys. Rev. D 51, R6608(1995).

[6] T. Hertog, Phys. Rev. D 74, 084008(2006).

[7] M. S. Volkov, and D. V. Galtsov, JETP. Lett. 50, 346(1989).

[8] P. Bizon, Phys. Rev. Lett. 64, 2844(1990).

[9] B. R. Greene, S. D. Mathur, and C. M. O’Neill, Phys. Rev. D 47, 2242(1993).

[10] J. E. Chase, Commun. Math. Phys. 19, 276(1970).

[11] Phys. Rev. Lett. 28, 452(1972).

[12] L. Heisenberg and S. Tsujikawa, Phys. Lett. B 780, 0370(2018).

[13] C. A. R. Herdeiro and E. Radu, Phys. Rev. Lett. 112, 221101(2014).

[14] V. Cardoso, I. P. Carucci, P. Pani and T. P. Sotiriou, Phys. Rev. D 88, 044056(2013).

[15] V. Cardoso, I. P. Carucci, P. Pani and T. P. Sotiriou, Phys. Rev. Lett. 111, 111101(2013).

[16] C. A. R. Herdeiro and E. Radu, Phys. Rev. Lett. 112, 221101(2014).

[17] C. Herdeiro, E. Radu and H. Runarsson, Class. Quant. Grav. 33, 154001(2016).

[18] C. Herdeiro and E.Radu, Class. Quant. Grav. 32, 144001(2015).

[19] T. Jacobson, Phys. Rev. Lett. 83, 2699(1999).

[20] M. W. Horbatsch and C. P. Burgess, JCAP. 1205, 010(2012).

[21] T. Torii, K. Maeda and M. Narita, Phys. Rev. D textbf64, 044007(2001).

[22] E. J. Copeland, M. Sami and S. Tsujikawa, Int. J. Mod. Phys. D 15, 1753(2006); T. P. Sotiriou and V. Faraoni, Rev. Mod. Phys. 82, 451(2010).

[23] L. Heisenberg, arXiv:1801.01523[gr-qc].

[24] S. W. Hawking, Nature 248, 30 (1974).

[25] S. W. Hawking, Commun. Math. Phys. 43, 199 (1975) Erratum: [Commun. Math. Phys. 46, 206 (1976)].

[26] T. Ghose, (Live Science, January 14, 2016).

[27] W. G. Unruh, Phys. Rev. D 14, 870 (1976).

[28] S. Shankaranarayanan, K. Srinivasan and T. Padmanabhan, Mod. Phys. Lett. A 16, 571(2001).

[29] K. Srinivasan and T. Padmanabhan, Phys. Rev. D 60, 024007(1999).

[30] E. C. Vagenas, Nuovo Cim. B 117, 899(2002).

[31] P. Kraus and F. Wilczek, Nucl. Phys. B 433, 403(1995).

[32] R. Kerner and R. B. Mann, Phys. Rev. D 73, 104010(2006).

[33] R. Kerner and R. B. Mann, Class. Quantum Grav. 25, 095014(2008).

[34] M. K. Parikh and F. Wilczek, Phys. Rev. Lett. 85, 5042 (2000).

[35] E. T. Akhmedov, V. Akhmedova and D. Singleton, Phys. Lett. B 642, 124 (2006).

[36] R. Kerner and R. B. Mann, Phys. Rev. D 73, 104010 (2006).

[37] M. Angheben, M. Nadalini, L. Vanzo and S. Zerbini, JHEP 0505, 014 (2005).

[38] D. Singleton and S. Wilburn, Phys. Rev. Lett. 107, 081102 (2011).

[39] I. Sakalli, K. Jusufi and A. Övgün, arXiv:1803.10583 [gr-qc].

[40] P. A. Gonzalez, A. Övgün, J. Saavedra and Y. Vasquez, Gen. Rel. Grav. 50, no. 6, 62 (2018).

[41] K. Jusufi, I. Sakalli and A. Övgün, Gen. Rel. Grav. 50, no. 1, 10 (2018).

[42] X. M. Kuang, J. Saavedra and A. Övgün, Eur. Phys. J. C 77, no. 9, 613 (2017).

[43] A. Övgün and I. Sakalli, Int. J. Theor. Phys. 57, no. 2, 322 (2018).

[44] K. Jusufi, A. Övgün and G. Apostolovska, Adv. High Energy Phys. 2017, 8798657 (2017).

[45] I. Sakalli and A. Ovgun, EPL 118, no. 6, 60006 (2017).

[46] K. Jusufi and A. Övgün, Int. J. Theor. Phys. 56, no. 6, 1725 (2017).

[47] A. Övgün, Adv. High Energy Phys. 2017, 1573904 (2017).

[48] I. Sakalli and A. Övgün, Eur. Phys. J. Plus 131, no. 6, 184 (2016).

[49] I. Sakalli, A. Övgün and K. Jusufi, Astrophys. Space Sci. 361, no. 10, 330 (2016).

[50] I. Sakalli and A. Övgün, J. Astrophys. Astron. 37, 21 (2016).

[51] K. Jusufi and A. Övgün, Astrophys. Space Sci. 361, no. 7, 207 (2016).

[52] A. Övgün and K. Jusufi, Eur. Phys. J. Plus 131, no. 5, 177 (2016).

[53] A. Övgün, Int. J. Theor. Phys. 55, no. 6, 2919 (2016).

[54] I. Sakalli and A. Övgün, Gen. Rel. Grav. 48, no. 1, 1 (2016). 
[55] I. Sakalli and A. Ovgun, Astrophys. Space Sci. 359, no. 1, 32 (2015).

[56] I. Sakalli and A. Ovgun, Eur. Phys. J. Plus 130, no. 6, 110 (2015).

[57] I. Sakalli and A. Ovgun, J. Exp. Theor. Phys. 121, no. 3, 404 (2015).

[58] I. Sakalli and A. Ovgun, EPL 110, no. 1, 10008 (2015).

[59] I. Sakalli, A. Ovgun and S. F. Mirekhtiary, Int. J. Geom. Meth. Mod. Phys. 11, no. 08, 1450074 (2014).

[60] M.Hossain Ali and K. Sultana, Int.J.Theor.Phys. 56, no.7, 2279-2292 (2017).

[61] M.Hossain Ali, Class.Quant.Grav. 24, 5849-5860 (2007).

[62] M.Hossain Ali, Int.J.Theor.Phys. 47, 2203-2217 (2008).

[63] M.Hossain Ali, Gen.Rel.Grav. 36, 1171-1181 (2004).

[64] H. Pasaoglu and I. Sakalli, Int. J. Theor. Phys. 48, 3517 (2009).

[65] I. Sakalli, M. Halilsoy and H. Pasaoglu, Int. J. Theor. Phys. 50, 3212 (2011).

[66] I. Sakalli, M. Halilsoy and H. Pasaoglu, Astrophys. Space Sci. 340, no. 1, 155 (2012).

[67] I. Sakalli, Astrophys. Space Sci. 340, 317 (2012).

[68] S. H. Mazharimousavi, I. Sakalli and M. Halilsoy, Phys. Lett. B 672, 177 (2009).

[69] I. A. Meitei, T. I. Singh, S. G. Devi, N. P. Devi and K. Y. Singh, Int. J. Mod. Phys. A 33, no. 12, 1850070 (2018).

[70] K. K. J. Rodrigue, M. Saleh, B. B. Thomas and T. C. Kofane, Gen. Rel. Grav. 50, no. 5, 52 (2018).

[71] Y. H. Ma, J. F. Chen and C. P. Sun, Nucl. Phys. B 931, 418 (2018).

[72] Y. H. Ma, Q. Y. Cai, H. Dong and C. P. Sun, EPL 122, no. 3, 30001 (2018).

[73] A. S. Sefiedgar and A. Ashrafinejad, EPL 119, no. 3, 30005 (2017).

[74] G. Gecim and Y. Sucu, Adv. High Energy Phys. 2018, 8728564 (2018).

[75] H. L. Li and S. R. Chen, Gen. Rel. Grav. 49, no. 10, 128 (2017).

[76] Z. W. Feng and S. Z. Yang, Int. J. Theor. Phys. 57, no. 3, 931 (2018).

[77] T. I. Singh, I. A. Meitei and K. Y. Singh, Int. J. Theor. Phys. 56, no. 8, 2640 (2017).

[78] S. Haldar, C. Corda and S. Chakraborty, Adv. High Energy Phys. 2018, 9851598 (2018).

[79] G. Gecim and Y. Sucu, Adv. High Energy Phys. 2018, 7031767 (2018).

[80] G. Q. Li and J. X. Mo, Gen. Rel. Grav. 49, no. 4, 57 (2017).

[81] G. Q. Li, Chin. Phys. C 41, no. 4, 045103 (2017).

[82] G. Gecim and Y. Sucu, Eur. Phys. J. Plus 132, no. 3, 105 (2017).

[83] W. Javed, G. Abbas and R. Ali, Eur. Phys. J. C 77, no. 5, 296 (2017).

[84] M. Rizwan and K. Saifullah, Gen. Rel. Grav. 48, no. 12, 163 (2016).

[85] M. Saleh, B. B. Thomas and T. C. Kofane, Front. Phys. (Beijing) 10, no. 5, 100401 (2015).

[86] H. Ding and W. b. Liu, Front. Phys. (Beijing) 6, 106 (2011).

[87] A. F.Ali, JHEP 1209, 067(2012).

[88] B. Majumder, Phys. Lett. B701, 384(2011).

[89] A. Bina, S. Jalalzadeh and A. Moslehi, Phys. Rev. D81, 023528(2010).

[90] P. Chen, and R. J. Adler, Nucl. Phys. Proc. Suppl. 124, 103(2003).

[91] R. J. Adler, P. Chen and D. I. Santiago, Gen. Relativ. Gravit. 33, 2101(2001).

[92] L. Xiang and X. Q. Wen, JHEP. 0910, 046(2009).

[93] W. Kim, E. J. Son and M. Yoon, JHEP. 0801, 035(2008).

[94] K. Nozari and S. H. Mehdipour, Europhys. Lett. 84, 20008(2008).

[95] A. Ovgun and K. Jusufi, Eur. Phys. J. Plus. 132, 298(2017).

[96] A. Ovgun, W. Javed and R. Ali, Advances in High Energy Physics, vol. 2018, Article ID 3131620, 11 pages, 2018.

[97] M. Sharif and W. Javed, Can. J. Phys. 90, 903(2012); ibid.

Gen. Relativ. Gravit. 45, 1051(2013); ibid. Can. J. Phys. 91, 43(2013); ibid. J. Exp. Theor. Phys. 115, 782(2012); ibid. Proceedings of the 3rd GalileoXu Guangqi Meeting, Int. J. Mod. Phys. [Conference Series], 23, 271(2013);ibid. Proceedings of the 13th Marcel Grossmann Meeting (Stockholm, 2012), World Scientific, 3, 1950(2015).

[98] M. Sharif and W. Javed, Eur. Phys. J. C72, 1997(2012).

[99] M. Sharif and W. Javed, J. Korean. Phys. Soc. 57, 217(2010).

[100] E. Papantonopoulos, Physics of Black Holes: A Guided Tour, (Springer, 25 Nov, 2008).

[101] E. Papantonopoulos, Modifications of Einstein's Theory of Gravity at Large Distances, (Springer International Publishing, 13 Nov, 2014)

[102] G. Gecim and Y. Sucu, Phys. Lett. B 773, 391(2017).

[103] Y. Sucu and N.Unal, Journal of Math. Phys. 48(2007)052503. 